\title{
Vidéoclip ou Psyché ranimée par le baiser de l’Amour
}

\author{
Claudine Potvin
}

\begin{abstract}
Videoclip or Psyche Revived By Cupid's Kiss
The text did not correspond to the image of it she had kept the angel breaks through the stone certainly supports the breast and ear in the same bound fastens on to the woman with her sex nonchalantly veiled whose arms extend the wings of the child... extremities of a base worked by the sculptor's chisel block doubly placed on the tiles of the gallery Mirna refuses the seduction of the white angel the scene of the kiss will not take place, she asserts in her disenchantment the murmur of art the heroine's doubt ... the real Mirna exists between the foil and the transparency... on the other side of fiction ... scraps of erotic discourse badly written, rehearsed and played the real Mirna ... loves herself describes brings to mind roses gardens the beauty of women and of the vegetation
\end{abstract}

Je veux que l'écriture s'approche de sa fraude s'aime malgré tout. L'écriture des mots appris après l'image. L'écriture des mots imprimés sur des gravures déjà là. L'écriture des mots compris sur toute représentation. ... Des mots de corps pleins la bouche. ... Ce désert d'eau, cette pluie qui persiste, mes pieds partout dans les petites flaques d'eau. ... Nous cherchons tous une oasis de sécheresse. (Madeleine Gagnon, Lueur)

le texte ne correspondait pas à l'image qu'elle en avait conservée l'ange franchit la pierre certes soutient le sein et l'oreille d'un même élan s'accroche à la femme au sexe nonchalamment voilé dont les bras prolongent les ailes de l'enfant une certaine décadence sur fond gris durcit le marbre des corps penchés sur le drap devenu inutile mais le prolongement des jambes fragiles et trop maigres tombe mal faux points d'appui entre le genou et le pied pendant, extrémités d'un socle travaillé par le ciseau du sculpteur bloc doublement posé sur les dalles de la galerie Mirna refuse la séduction de l'ange blanc la scène du baiser 
n'aura jamais lieu, affirme-t-elle dans son désenchantement le murmure de l'art le doute de l'héroïne

lentille grossissante loupe de nuit une femme cuirassée la peau des autres s'accumule couche par couche sur le revers de la sienne une femme se brosse la peau s'épile se frotte s'écaille se sèche se filme sous l'eau s'écume se caresse se représente en train de bouge s'appuie les mains se soulève regarde la photographie d'un bel ange évanoui endormi dans les bras de Psyché

Mirna épuisée out of print sans empreinte les femmes ne laissent pas de traces, pense-t-elle les sentiers empruntés prêts temporaires incertains images puritaines pas de femmes lits de femmes se résument parfois à peu d'images une traînée s'effondrant dans le désert en poudre en lèvres en larmes balancer l'enfance aux portes des villes bondées de femmes folles de musées de marbre poli de formes amollies de centres cibles

Mirna refuse le jour l'étalement du temps sur la peau des bêtes émaillées archéologue du corps un mot résorberait l'angoisse ça n'avait plus d'importance, songeait-elle pour commencer un tableau, il faut qu'il y ait un grand vide au milieu

l'ange exterminateur passe dans le reflet de la baignoire la femme se glisse au milieu des bulles passoires de nuit laissez rêver la couleur de la pellicule un train siffle près de la route une voie ferrée en perte de vitesse de chaque côté du corps deux longs bras comme des ailes d'ange

il pleut dans le bain de Mirna le tourbillon bouillonne au creux de son ventre ballonné le sexe écrin enveloppé sous l'onde soulevé par la vague un sexe pleureur en train de boire pulsions de survie Mirna fredonne le texte imprimé sur le carrelage rose refuse l'écriture du dehors se concentre sur la métaphore transparente ne se passe rien dans la soirée de Mirna

la vie n'avance plus beau fixe désire la tempête le vent chavire les voiles déchire la toile blanche ensoleillée assoupie le baiser la moiteur de la tempe étrangère la rive l'accotement la $\mathrm{p}(\mathrm{l})$ age les pins gommés l'éclair/si...

le récit de Mirna s'amorce toujours au bain l'heure de l'observation du 
contentement ou du mécontentement ce soir c'est l'entre-deux l'ouverture des cuisses laisse glisser le savon à la glycérine s'efface dans les plis de l'eau qui forment une jupe ou une ombrelle turquoise sur le grand corps effiloché se faufile entre les doigts minaudiers l'eau s'amuse autour des reins de la nymphe parlante descend le long de la colonne selon l'angle du corps qui s'offre s'abandonne remonte les cils les paupières les sourcils l'oeil de la nuit reproduit un miroir

pour la femme l'ombre de sa beauté sur la surface brumeuse de son rêve renvoie l'image flottante d'une robe de satin texture de l'icône grecque drapée d'ocre et de rouge dans les sueurs des bains publics qui est-elle l'ange du plateau l'Ang/elle qui joue la scène pornographique de ses fantasmes enfantins l'aile du désir le regard d'une enfant sourde et muette

il pleut elle entend le son de la pluie la soirée humide sur le carreau les vapeurs d'eau se trempe se mouille d'y penser Pénélope attendant Ulysse Pénélope contemplant la chute de l'eau sur la tapisserie le jeu des laines le mélange des fils l'amour des mains vagabondes les généreuses filles bleues dans l'océan indien les pacifiques la joie sa joie la fenêtre suinte

Mirna se touche tout partout sentir la chair s'amonceler sous la prise reconnaître les marques de l'âge identifier les cernes effeuiller les épisodes saisir le vécu au bout des orteils sentir des coulées de sable de sel sous la couverture

Mirna avale par petites gorgées le café noir qu'il vient de lui apporter fort comme elle l'aime a renversé une goutte sur la page blanche du livre qu'elle feuillette entre deux chapitres le café n'a effacé aucun mot le récit de Mirna semble intact se réchauffe encore plus sent son visage inondé la rougeur transpercer le haut de son corps des rouges au coeur, penset-elle sur le côté gauche les traces de la tasse ont dessiné une série de pistes le plaisir des explorations imprévues espaces logés rangés courbes en diagonale géographies clandestines monts de Vénus au milieu des déserts féminins effets de serre tropicale sous la pression de désir des plantes poussent

l'huile du bain a laissé une épaisse ligne noire sur l'émail Mirna s'amuse à la défaire avec la pointe finement aiguisée de son crayon 
inscrivant malhabile les mots du silence de la solitude le temps ne se compte plus les minutes les heures passent voguent le répit n'existe plus que pour elle en dehors de son histoire, Mirna reproduit le son des gouttes de pluie dans le lit de la rivière séchée repense les images de l'enfance le corps de la mère et du père couchés l'un sur l'autre au milieu des bois le déplacement des rides la fausse tendresse de l'ivresse la complainte du froid des lèvres glacées le torse creux de son premier amant dans la forêt les arbres drus de la plaine les projections de Mirna forment une immense boule de savon le signet crève la nuit perce la pensée l'attente la réflexion de l'eau dans le cerveau de Mirna en ébullition se rit des bois verts et de la lumière des avenues marbrées de la ville

le rire de Mirna est contagieux à la scène sur le plateau dans la foule il dégringole et forme une mousse d'herbe où il fait bon s'étendre Mirna verte Mirna sonore elle réchauffe la nuit se sait s'écrit alors d'une mémoire à l'autre

Mirna sortie du bain s'enroule dans la serviette rose saumon avant de se contempler longuement dans la glace vénitienne refuse le toucher cette fois feint de se découvrir interroge le miroir dis-moi, qui est la plus belle celle de l'autre côté ou Mirna la vraie derrière l'écran se cache une femme masquée vieillie presque heureuse la chair rouillée la grandmère la tante la soeur femmes d'ailleurs images insérées dans les premières leçons apprentissages rigides différent le corps se plie sous le coup du manuel Mirna la vraie existe-t-elle entre le tain et la tranparence

de l'autre côté de la fiction, Mirna se raconte un texte délavé embué ancré entre l'index et le pouce là où la chaleur s'insinue sous la couche de l'épiderme là où survivre revient à se grandir se planter sur les talons pour voir le sexe son sexe dans la fente d'une serviette avec laquelle on éponge les miettes des discours érotiques mal écrits mal répétés mal joués

Mirna la vraie sait bien qu'elle est la plus belle quand elle se regarde dans le miroir s'admire se plaît se flatte éprouve de l'affection pour se délice se savoure gâteau se chante se poème se chatouille hume profondément le corps plein de sa poitrine serre les poings au moment de l'orgasme retient la chevelure brune les éclaircies jetées ici et là sur la peau veloutée s'aime se décrit se remémore des roses des jardins la beauté des femmes et de la végétation 